\title{
As abelhas, os serviços ecossistêmicos e o Código Florestal Brasileiro
}

\author{
Vera Lucia Imperatriz-Fonseca ${ }^{1,2,4}$ \& Patrícia Nunes-Silva ${ }^{3}$ \\ ${ }^{1}$ Instituto de Estudos Avançados, Universidade de São Paulo - USP \\ ${ }^{2}$ Universidade Federal Rural do Semiárido - UFERSA \\ ${ }^{3}$ Pós-graduação em Entomologia, Faculdade de Filosofia, Ciências e Letras de Ribeirão Preto, \\ Universidade de São Paulo - USP \\ ${ }^{4}$ Autor para correspondência: Vera Lucia Imperatriz Fonseca, e-mail: vlifonse@ib.usp.br
}

IMPERATRIZ-FONSECA, V.L. \& NUNES-SILVA, P. Bees, ecosystem services and the Brazilian Forest Code. Biota Neotrop. 10(4): http://www.biotaneotropica.org.br/v10n4/en/abstract?article+bn00910042010.

\begin{abstract}
Bees are considered the main pollinators in natural and agricultural environments. This ecosystem service is essential to the maintenance of wild plant populations and to food production on agricultural environments and it's threatened in many regions of the world. Deforestation is pointed out as one of the main causes because it affects bee populations. Conservation of forests is necessary for the maintenance of bee populations and pollination services on agricultural landscapes.
\end{abstract}

Keywords: bees, ecosystem services, Brazilian Forest Code.

IMPERATRIZ-FONSECA, V.L. \& NUNES-SILVA, P. As abelhas, os serviços ecossistêmicos e o Código Florestal Brasileiro. Biota Neotrop. 10(4): http://www.biotaneotropica.org.br/v10n4/pt/abstract?article+bn00910042010.

Resumo: As abelhas são consideradas os principais polinizadores em ambientes naturais e agrícolas. Esse serviço ecossistêmico é essencial para a manutenção das populações selvagens de plantas e para a produção de alimento nos ambientes agrícolas e está ameaçado em várias regiões do mundo. O desmatamento é uma das causas principais porque ele afeta as populações de abelhas. A conservação das florestas é necessária para a manutenção das populações de abelhas e da polinização nas paisagens agrícolas.

Palavras-chave: abelhas, serviços ecossistêmicos, Código Florestal Brasileiro. 


\section{Introdução}

A grande mudança em relação à aceitação do conceito de serviços ecossistêmicos surgiu quando em 1997, Constanza et al. (1997) publicaram um famoso estudo ( $O$ valor dos serviços ecossistêmicos do mundo e o capital natural) na revista Nature, uma das mais prestigiadas da atualidade, dando um valor para cada um dos 17 serviços ecossistêmicos. Este é o segundo trabalho mais citado da revista Nature na área de meio ambiente nos últimos 10 anos. Uma vez que seria possível quantificar quanto vale a natureza se ela precisasse ser reconstruída, o capital natural passa a ser um ponto central nas discussões relacionadas ao meio ambiente e sua utilização. Outro termo utilizado é o de serviços ambientais: no Brasil já há leis que apóiam o pagamento pelos serviços ambientais de manutenção de mananciais, por exemplo no Estado de São Paulo. A Economia Ecológica desenvolveu o conceito de capital natural (Constanza et al. 1997) para tratar dos recursos não renováveis, os renováveis e os serviços dos ecossistemas para os cientistas sociais.

Há algumas definições para os serviços dos ecossistemas:

- As condições e processos através dos quais os ecossistemas naturais e as espécies que os compõem sustentam a vida humana (Daily 1997).

- Os benefícios das populações humanas derivam, direta ou indiretamente, das funções dos ecossistemas (Constanza et al. 1997).

- Os benefícios que os homens obtêm dos ecossistemas (Avaliação do Milênio 2005).

Em 1920, éramos dois bilhões de habitantes na Terra, agora somos mais de 6,5 bilhões de habitantes, que necessitam de alimento, fibras, água, entre outros produtos e serviços. No ano 2000, o Secretário da Organização das Nações Unidas, Kofi Annan, encomendou um grande estudo para avaliar qual é a importância do Meio Ambiente para o bem estar do homem. A base deste trabalho foi o serviço ecossistêmico. A análise, que se iniciou em 2001 e foi até 2005, foi feita por 1300 cientistas além de tomadores de decisão, coordenadores, etc., e ficou conhecida como a Avaliação do Milênio (Millennium Ecosystem Assesment 2005). Muitos documentos foram então produzidos, inclusive disponíveis em português (por exemplo, Vivendo além de nossos meios - o Capital Natural e o bem estar humano).

Perdemos $35 \%$ dos manguezais, $40 \%$ das florestas e $50 \%$ das áreas alagadas. A pesca foi super explorada, de modo que os estoques de peixes são $80 \%$ menores. Além disso, $60 \%$ dos serviços dos ecossistemas foram degradados nos últimos 50 anos. A perda de diversidade é de 100 a 1000 vezes maior do que em outros séculos (Röckstrom et al. 2009). A área cultivada do planeta cobriu $25 \%$ da superfície da Terra. O uso da terra é disputado: são muito focalizados os limites entre agricultura e conservação, de tal modo que os novos mapas de preservação da Terra são baseados nos serviços ecossistêmicos (Turner et al. 2007).

\section{Resultados e Discussão}

As abelhas e plantas com flores evoluíram juntas há muitos milhões de anos, de modo que existem muitas especializações nas várias espécies de abelhas para a coleta de alimento nas flores. As plantas se beneficiam das visitas das abelhas: estas voam de flor em flor, quando ficam aderidos ao corpo das abelhas os grãos de pólen (gametas masculinos) da flor visitada, que poderão ser depositados no estigma (gameta feminino) da próxima flor, processo chamado de polinização. Assim, as abelhas prestam um serviço às plantas, a polinização e a fertilização cruzada (Figura 1).

A polinização é considerada um serviço ecossistêmico regulatório. Ela é importante para a produção de alimentos (flores bem polinizadas produzem frutos de melhor qualidade, peso e sementes em maior

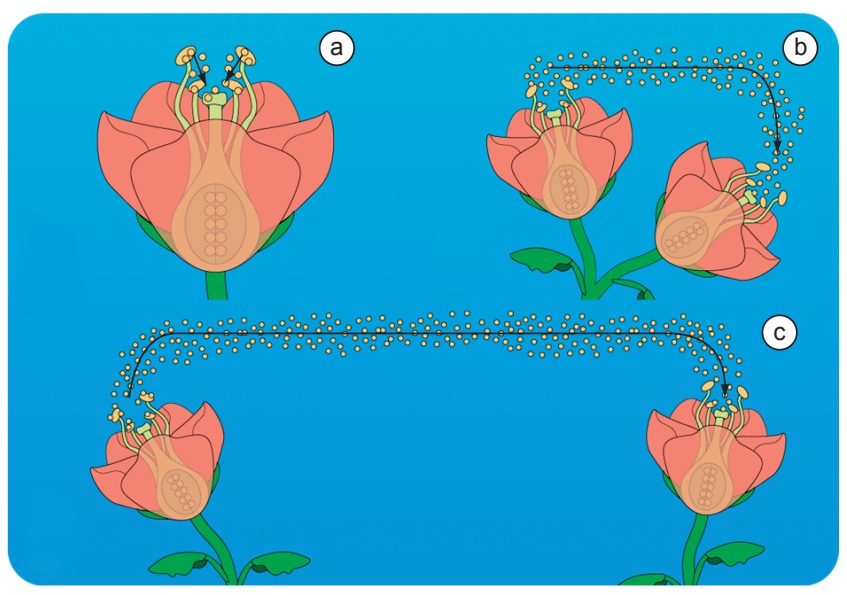

Figura 1. Esquema de autopolinização (a- mesma flor; b- diferentes flores, mesma planta) e de polinização cruzada (c- flores de plantas diferentes). Créditos: Bruno Nunes.

Figure 1. Self pollination (a- same flower; b- different flower, same plant) and cross pollination (c - flowers from different plants). Credits: Bruno Nunes.

número (Ricketts et al. 2008)) de biocombustíveis (Rizzardo et al. 2008, Durán et al. 2010), e principalmente para a manutenção da biodiversidade em áreas naturais, um serviço de valor inestimável. As árvores tropicais dependem em $90 \%$ de animais polinizadores (Bawa 1990). A produção de frutos está na base da cadeia alimentar, sendo de fundamental importância para o equilíbrio dos ecossistemas.

A biodiversidade de abelhas no mundo todo é muito grande, são conhecidas cerca de 20.000 espécies (ITIS 2010). Destas, a grande maioria tem hábitos solitários, e cerca de 1000 espécies são sociais.

O Brasil é muito rico em espécies de abelhas. Só no Estado de São Paulo temos mais de 720 espécies (Pedro \& Camargo 1999)! O que elas têm em comum, entretanto, é que dependem das flores para ali obterem o seu alimento e o de sua prole: o pólen das flores é a fonte de proteínas, o néctar a fonte de açúcares.

Essa diversidade de abelhas interage com um grande número de espécies de plantas. Por exemplo, em um estudo realizado em uma área do Agreste pernambucano por Milet-Pinheiro \& Schlindwein (2008) 79 espécies de abelhas foram coletadas em 87 espécies de plantas. Outro estudo feito por Silva et al. (2010) com quatro espécies de Xylocopa (Xylocopa frontalis, X. grisescens, X. hirsutissima e $X$. suspecta) em quatro fragmentos de Cerrado sentido restrito verificaram que essas espécies de abelhas visitam 30 espécies de plantas, sendo 26 delas nativas desse bioma.

Avaliações recentes baseadas nos bancos de dados da FAO (Food and Agricultural Organization) confirmam que 33\% da alimentação humana depende em algum grau de plantas cultivadas polinizadas muitas vezes pelas abelhas (Klein et al. 2007). Muitos associam as abelhas à espécie Apis mellifera, também conhecida como abelha doméstica e atualmente existente em todos os continentes, pois foi introduzida pelos colonizadores nas Américas e na Austrália. Elas são polinizadores fundamentais para a agricultura, assim como poucos outros que também são criados em escala comercial, e o valor deste serviço da polinização agrícola foi estimado como sendo de $9,5 \%$ do valor da agricultura em 2005, ou 153 bilhões de Euros (Gallai et al. 2009). As análises econômicas do valor da polinização e as estimativas de seu impacto na agricultura de países tropicais não pararam por aí. 
Dentro desta abordagem mais ampla da ligação entre agricultura e serviços da polinização, foram realizadas outras análises globais, utilizando-se a base de dados de culturas agrícolas da FAO. Dados das exportações de alimentos entre 1961-2006 (Aizen et al. 2009) evidenciaram que o plantio de culturas agrícolas que dependem de polinizadores está crescendo mais rapidamente que o daquelas que não dependem, tanto em países desenvolvidos como nos em desenvolvimento. A demanda crescente do uso de polinizadores para maior rendimento econômico foi evidenciada, para países em desenvolvimento, para frutas e verduras (Aizen et al. 2008). As projeções realizadas foram de que a apicultura está crescendo em ritmo mais lento do que a necessidade dos serviços ecossistêmicos manejáveis que ela presta (Aizen \& Harder 2009).

De acordo com a base de dados da FAO, os países em desenvolvimento compreendem os países africanos e latinoamericanos, assim como a maioria dos países do Sudoeste da Ásia, China e Índia. O mundo desenvolvido compreende os países europeus, os Estados Unidos, Canadá, Austrália e Nova Zelândia. A agricultura nos países em desenvolvimento representa mais de dois terços da agricultura mundial e é $50 \%$ mais dependente da polinização que a agricultura dos países desenvolvidos (Aizen et al. 2008). No caso das culturas dependentes de polinização, na ausência de polinizadores seria necessário plantar uma área seis vezes maior nos países em desenvolvimento para obter a mesma produtividade que os países desenvolvidos apresentam, dado este da maior importância quando o assunto é o uso da terra, visto que essa situação pode acelerar o desmatamento e intensificar a pressão que os fragmentos florestais já sofrem para aumentar a área de plantio a fim de compensar a baixa produtividade (Aizen et al. 2009).

Em 1961 a produção agrícola era semelhante nos países desenvolvidos e em desenvolvimento; entretanto, em 2006 esta produção era 2,2 vezes maior nos países em desenvolvimento. De 1961 a 2002, a dependência dos polinizadores cresceu 50\% nos países desenvolvidos (com um déficit de polinização de 3 a $5 \%$ ) e $62 \%$ nos países em desenvolvimento (com déficit de polinização de cerca de $8 \%$ ). No período de 1961 a 2006 a área cultivada aumentou $25 \%$. Em 1961 a área cultivada nos países em desenvolvimento era 38\% maior do que nos países desenvolvidos, mas esta diferença passou para $130 \%$ em 2006. O valor dos serviços da polinização na América do Sul é de 11,6 bilhões de euros por ano (Potts et al. 2010). Dessa forma, considerando o aumento da área cultivada e da dependência da agricultura nos serviços de polinização e o valor desse serviço, é necessário tornar as paisagens agrícolas capazes de manter os polinizadores. Uma forma de manter os polinizadores é preservar os locais de nidificação das abelhas, como troncos apodrecidos e barrancos, no caso de abelhas solitárias (Alves-dos-Santos 2009, Freitas \& Alves 2009)e árvores com ocos, no caso das abelhas sociais (Cortopassi-Laurino et al. 2009) e isso é possível conservando e melhorando as condições dos fragmentos de matas.

Mencionamos aqui alguns dados de pesquisas recentes efetuadas no Brasil que evidenciam o valor econômico dos polinizadores. Mesmo em espécies onde ocorre a auto-fecundação, e, portanto não há obrigatoriedade da atuação de agentes polinizadores, por exemplo café, canola e soja, que são autopolinizados, há um aumento considerável da produção se a cultura tiver a visita de abelhas polinizadoras (Veddeler et al. 2008). No café, estudos realizados em várias partes do mundo evidenciam um aumento de produtividade de 14\% (De Marco \& Coelho 2004) a 50\% (Ricketts et al. 2008) dependendo da paisagem do entorno ser amigável aos polinizadores, isto é, de haver nas proximidades condições para os polinizadores viverem, construírem seus ninhos e se reproduzirem. No caso da canola, estudos recentes (Durán et al. 2010) mostram um aumento de $53 \%$ nas plantas polinizadas. No caso da mamona (Rizzardo et al. 2008), embora o nectário seja extrafloral, os cultivos com abelhas apresentaram um aumento de 5\% na produtividade, melhor qualidade do óleo obtido e a produção de mel de boa qualidade como insumo complementar. Para as árvores da floresta, podemos mencionar que sem abelhas pequenas o cupuaçu não frutifica (Gribel 2008), que a castanha do Brasil depende de espécies de abelhas grandes e fortes como polinizadoras (Freitas \& Cavalcante 2008) e o açaí é totalmente dependente de polinizadores para produzir seus frutos (Venturieri 2005), de excelente valor comercial. Mesmo o dendê depende de polinização por besouros (Kevan 1995).

Por estes motivos aqui mencionados, a manutenção de área natural nas propriedades agrícolas é um seguro para a maior produção agrícola. Uma vez que estes dados dos serviços ambientais estão sendo divulgados e avaliados, será uma enorme perda para os proprietários rurais desmatarem mais as suas propriedades. Devemos mencionar aqui mais um fator de risco, as projeções de alterações climáticas severas para os próximos anos. Os ambientes mais conservados manterão maior resiliência, isto é, a capacidade de regeneração. Recuperação ambiental é o melhor seguro. É tempo de mudar de paradigma e focalizar as paisagens rurais amigáveis aos serviços ecossistêmicos.

\section{Referências Bibliográficas}

AIZEN, M.A. \& HARDER, L.D. 2009. The global stock of domesticated honey bees is growing slower than agricultural demand for pollination. Curr. Biol. 19:915-918.

AIZEN, M.A., GARIBALDI, L.A., CUNNINGHAM, S.A. \& KLEIN, A.M. 2008. Long-term global trends in crop yield and production reveal no current pollination shortage but increasing pollinator dependency. Curr. Biol. 18:1572-1575.

AIZEN, M.A., GARIBALDI, L.A., CUNNINGHAM, S.A. \& KLEIN, A.M. 2009. How much does agriculture depend on pollinators? Lessons from long-term trends in crop production. Ann. Bot. 103:1579-1588.

ALVES-DOS-SANTOS, I. 2009. Por que preservar troncos apodrecidos e barrancos em sua propriedade? Mensag. Doce $100 \mathrm{http}: / / \mathrm{www} . a p a c a m e$. org.br/mensagemdoce/100/artigo.htm (último acesso em 28/09/2010).

BAWA, K. 1990. Plant-pollinator interactions in tropical rain-forests. Ann. Rev. Ecol. Syst. 21:399-422.

CONSTANZA, R., D’ARGE, R., GROOT, R., FARBERK, S., GRASSO, M., HANNON, B., LIMBURG, K., NAEEM, S., PARUELO, J., RASKIN, R.G., SUTTON, P. \& VAN DEN BELT, M. 1997. The value of the world's ecosystem services and natural capital. Nature 387:253-260.

CORTOPASSI-LAURINO, M., ARAUJO, D.A. \& IMPERATRIZ-FONSECA, V.L. 2009. Árvores neotropicais, recursos importantes para a nidificação de abelhas sem ferrão (Apidae, Meliponini). Mensagem Doce 100, http:// www.apacame.org.br/mensagemdoce/100/artigo4.htm (último acesso em 28/09/2010)

DAILY, G.C. 1997. Nature's services: Societal Dependence on Natural Ecosystems. Island Press, Washington, DC.

DE MARCO, P. \& COELHO, F.M. 2004. Services performed by the ecosystem: forest remnants influence agricultural cultures' pollination and production. Biodivers. Conserv. 13:1245-1255.

DURÁN, X.A., UllOA, R.B., CARRILlO, J.A., CONTRERAS, J.L. \& BASTIDAS, M.T. 2010. Evaluation of yield component traits of honeybee pollinated (Apis mellifera L.) Rapeseed canola (Brassica napus 1.). Chil. J. Agr. Res. 70:309-314.

FREITAS, B.M. \& CAVALCANTE, M.C. 2008. Visitantes florais e polinização da castanha do Brasil (Bertholletia excelsa) em cultivo comercial na Floresta Amazônica. In Anais do VIII Encontro sobre Abelhas, FUNPEC, Ribeirão Preto, p.58-66.

FREITAS, B. \& ALVES, J.E. 2009. Importância da disponibilidade de locais para nidificação de abelhas na polinização agrícola: o caso das mamangavas de toco. Mensag. Doce 100. http://www.apacame.org.br/ mensagemdoce/100/artigo2.htm (último acesso em 28/09/2010). 
GALlAI, N., SAlLES, J.M., SETTELE, J. \& VAISSIERE, B. 2009. Economic valuation of the vulnerability of world agriculture con- fronted with pollinator decline. Ecol. Econ. 68:810-821.

GRIBEL, R. 2008. Pollination ecology and pollinator management in cupuassu (Theobroma grandiflorum Willd. Ex Spreng. Schum., Sterculiaceae), an amazonian fruittree of promising economic importance. In Pollinators management in Brazil (C.A.B. Alvarez \& M. Landeiro, org.). Ministério de Meio Ambiente, Brasília, p.14-17.

INTERAGENCY TAXONOMIC INFORMATION SYSTEM - ITIS. Catalogue of life: 2010 annual checklist. 2010. http://www.catalogueoflife.org/annualchecklist/2010/details/database/id/67 (último acesso em 28/09/2010).

KEVAN, P.G. 1995. Insect pollination of economically important plants of tropical and subtropical Asia. In The Asiatic hive bee: apiculture, biology, and role in sustainable development in tropical and subtropical Asia (P.G. Kevan, ed.). Enviroquest, Cambridge, Canada, p.129-133.

KLEIN, A.M., VAISSIERE, B.E., CANE, J.H., STEFFAN-DEWENTER, I., CUNNINGHAM, S.A., KREMEN, C. \& TSCHARNTKE, T. 2007. Importance of pollinators in changing landscapes for world crops. Proc. Roy. Soc. B-Biol. Sci. 274:303-313.

MILET-PINHEIRO, P. \& SCHLINDWEIN, C. 2008. Comunidade de abelhas (Hymenoptera, Apoidea) e plantas em uma área do Agreste pernambucano, Brasil. Rev. Bras. Entomol. 52(4):625-636.

MILLENNIUM ECOSYSTEM ASSESSMENT. AVALIAÇÃO DO MILÊNIO. 2005. http://www.maweb.org/en/index.aspx (último acesso em 28/09/2010).

PEDRO, S.R.M. \& CAMARGO, J.M.F. 1999. Apoidea Apiformes. In Biodiversidade do Estado de São Paulo Invertebrados Terrestres. (C.A. Joly, C.E.M. Bicudo, C.R.F. Brandão \& E.M. Cancello, ed.). São Paulo, v.5, p.193-211.

POTTS, S.G., BIESMEIJER, J.C., KREMEN, C., NEUMANN, P., SCHWEIGER, O. \& KUNIN, W.E. 2010. Global pollinator declines: trends, impacts and drivers. Trends Ecol. Evol. 25:345-353.
RICKETTS, T., REGETZ, J., STEFFAN-DEWENTER, I., CUNNINGHAM, S.A., KREMEN, C., BOGDANSKI, A., GEMMIL-HERREN, B., GREENLEAF, S.S., KLEIN, A.M., MAYFIELD, M.M., MORANDIN, L.A., OCHIENG, A. \& VIANA, B.F. 2008. Landscape effects on crop pollination services: are there general patterns? Ecol. Lett. 11:499-515.

RIZZARDO, R.A.G., FREITAS, B.M., MILFONT, M.O. \& SILVA, E.M.S. 2008. A polinização de culturas agrícolas com potencial para produção de biodiesel: um estudo de caso com a mamona (Ricinus communis L.). In Anais do VIII Encontro Sobre Abelhas, FUNPEC, Ribeirão Preto, p.293-299.

RÖCKSTROM, J., STEFFEN, W., NOONE, K., PERSSON, Å., CHAPIN, F.S., LAMBIN, E.F., LENTON, T.M., SCHEFFER, M., FOLKE, C., SCHELLNHUBER, H.J., NYKVIST, B., WIT, C.A., HUGHES, T., VAN DER LEEUW, S., RODHE, H., SÖRLIN, S., SNYDER, P.K., COSTANZA, R., SVEDIN, U., FALKENMARK, M., KARLBERG, L., CORELL, R.W., FABRY, V.J., HANSEN, J., WALKER, B., LIVERMAN, D., RICHARDSON, L. CRUTZEN, P. \& FOLEY, J.A. 2009. A safe operating space for humanity. Nature 461:472-475.

SILVA, C.I., MELLO, M.A.R. \& OLIVEIRA, P.O. 2010. A palinologia como uma ferramenta importante nos estudos das interações entre Xylocopa spp. e plantas no Cerrado. In Anais do IX Encontro Sobre Abelhas, FUNPEC, Ribeirão Preto, p.72-79.

TURNER, W.R., BRANDON, K., BROOKS, T.M., COSTANZA, R., FONSECA, G.B. \& PORTELA, R. 2007. Global Conservation of Biodiversity and ecosystem services. Bioscience 57(10):868-873

VEDDELER, D., OLSCHEWSKI, R., TSCHARNTKE, T. \& KLEIN, A.M. 2008. The contribution of non managed social bees to coffee production: new insights based on farm-scale yield data. Agroforestry Syst. 73:109-114.

VENTURIERI, G.C., RODRIGUES, S.T. \& PEREIRA, C.A.B. 2005. As abelhas e as flores do açaizeiro (Euterpe oleracea Mart. - Arecaceae). Mensagem Doce 80:32-33. 